\title{
COVID-19 wave 4 in Western Cape Province, South Africa: Fewer hospitalisations, but new challenges for a depleted workforce
}

With the announcement of a new SARS-CoV-2 variant of concern containing 32 mutations to the spike protein, South Africa (SA) braced for another December of high rates of hospitalisations, oxygen utilisation and mortality. ${ }^{[1]}$ Omicron has lived up to expectations for high transmission and immune escape, with a reproduction number just over 2.5 recorded in Western Cape Province by December 2021. ${ }^{[2]}$ However, the public breathed a sigh of relief when early Gauteng data hinted at less severe disease. As of 20 December 2021, wave 4 in the Western Cape has fit a similar pattern, with high case rates and growing reinfections but much lower rates of hospitalisation, oxygen and high-care utilisation, and mortality in comparison with prior COVID-19 waves (Fig. 1). ${ }^{[2]}$ Despite the number of daily new diagnoses nearly reaching the wave 3 peak, hospitalisation rates remain a third of prior waves (6\% of all cases in wave $4 \mathrm{v} .18 \%$ in wave 1), while deaths and oxygen utilisation have not risen above inter-wave troughs (Fig. 1). ${ }^{[2]}$

While it is too early to fully assess reduction in disease severity in wave $4 \mathrm{v}$. earlier waves, as severe outcomes take time to occur, it is likely that a large contribution to the experience of high case numbers with less severe disease is a combination of immune escape by the Omicron variant and high rates of population immunity from prior infection and/or vaccination. In the Western Cape, $46 \%$ of the adult population is fully vaccinated, while $66 \%$ of those aged $\geq 60$ years are fully vaccinated. ${ }^{[3]}$ What's more, COVID-19 seroprevalence due to prior infection after the second wave was $\sim 50 \%$ across the Western Cape and nearly $70 \%$ in some areas, and probably rose by $\sim 20$ percentage points during the third Delta-driven wave. ${ }^{[4]}$ Although prior infection and/or vaccination are not preventing Omicron infection, immunity continues to protect most individuals against severe disease.

However, just because hospitals are not flooded with COVID-19 admissions, it does not mean that the healthcare system will cope with high volumes of mild disease, and mitigation of Omicron waves across the world will need to address this. Since 28 November
2021, in the public sector, 12793 COVID-19 cases have been diagnosed in the Western Cape from 42473 COVID-19 tests, with only $6 \%$ of those patients being hospitalised (analysis by the Epidemiology and Surveillance subdirectorate, Health Intelligence, Western Cape Government: Health - unpublished data, 19 December 2021 (WCGH)). Of the 5682 cases diagnosed at primary healthcare facilities, only $48(<1 \%)$ have been admitted to hospital. In fact, among all wave 4 admissions to date, only $6 \%$ were tested at primary healthcare facilities. Nearly all hospitalised cases $(93 \%)$ were first tested at hospital on the day of admission, suggesting that those with severe COVID-19 are seeking care only when they are very ill (WCGH). Essentially, primary healthcare services risk being swamped with the assessment and diagnosis of mild COVID-19 disease, as has occurred in the Western Cape, for arguably very little benefit to the patient or the public. No antiviral treatment is available in SA for mild COVID-19 disease. Likewise, severe COVID-19 patients are not presenting prior to admission, thereby negating any potential case management benefits of early diagnosis. Furthermore, case finding is not blunting community transmission rates. Testing is biased towards symptomatic cases, but a high proportion of COVID-19 cases are asymptomatic, and even in symptomatic cases, infectiousness starts before symptom onset. ${ }^{[5]}$ Even testing of symptomatic cases is far from universal, compounded by suboptimal SARS-CoV-2 test sensitivity, leading to many false-negative results. ${ }^{[6.8]}$ Seropositivity rates seen across multiple provinces suggest that as few as 1 in 10 cases might be being diagnosed..$^{[9]}$ While individual knowledge of one's diagnosis may enable one to isolate and protect others, since the vast majority of COVID-19 cases go undetected, and most COVID19 contacts occur in the context of a case that has not been identified in the first place, the public health impact of testing what is effectively a minority of total cases is negligible. Outpatient COVID-19 testing does not decrease community transmission, nor does it offer any benefit to individual clinical management, aside from a definitive diagnosis.

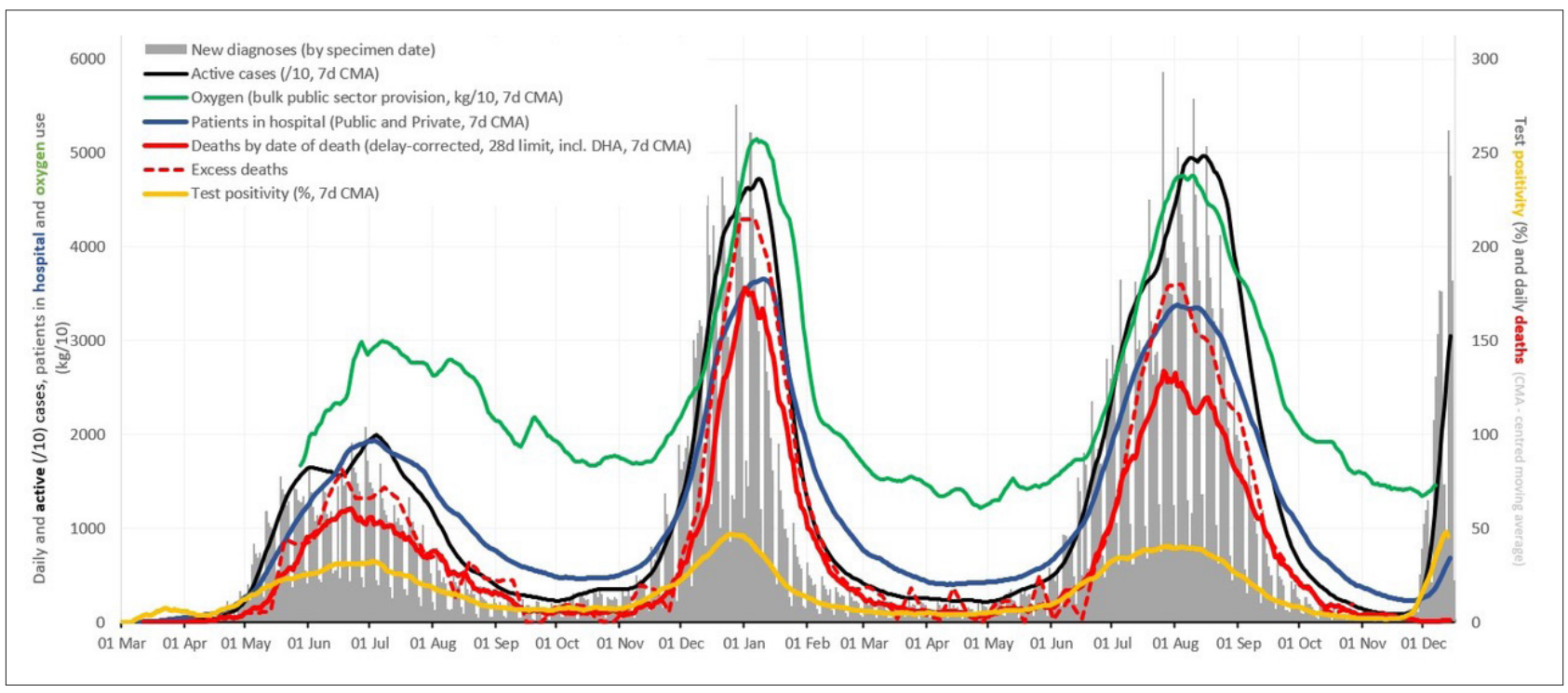

Fig. 1. Number of daily COVID-19 cases by specimen date and 7-day moving average of active cases, bulk oxygen consumption, current patients in hospital, COVID-19 deaths (by date of death) including linked deaths from the Department of Home Affairs, excess deaths and COVID-19 test positivity. (CMA = centred moving average.) 
Concern about being able to meet testing demand and the need to rather promote community mitigation measures has been raised in other jurisdictions such as the UK SAGE. ${ }^{[10]}$

Attempting to meet the service and testing demand of mild COVID19 cases is deeply damaging to all other primary healthcare services, as limited resources are diverted from usual operations towards assessing and testing the abundance of mild cases. Wave 4 is disproportionately affecting primary healthcare services, given that it is so far dominated by milder disease. As a result, fewer staff are available to attend to patients acutely ill with non-COVID-19 disease. Tuberculosis diagnosis is down by a third. Chronic HIV and non-communicable disease patients' prescriptions are being re-written, often without patient assessment, so that limited clinical staff can attend to the glut of mild COVID-19 patients. Anecdotally, after 18 months of health service challenges, many chronic disease patients are presenting with severe morbidity that could have been prevented - late-diagnosed metastatic cancers, uncontrolled blood glucose resulting in end-organ damage, opportunistic infections from advanced HIV.

Testing of mild COVID-19 cases is one example of how we have prioritised COVID-19 over other health conditions, and this comes at a cost. While this cost, which included de-escalation of other health services such as delaying elective surgery, was justifiable in previous waves when severe COVID-19 threatened to overwhelm acute hospital services, it is questionable in the context of less severe disease. To preserve the integrity of primary healthcare services during COVID-19 surges, testing strategies must change. Options could include ramping up testing stations that are delinked from primary healthcare facilities in which public sector patients with mild disease could obtain a COVID-19 test, affordable home-based self-testing, or, if neither of these are feasible, restricted testing to severe COVID-19 patients requiring hospital admission. Owing to affordability and speed of diagnosis, antigen tests should be prioritised, especially for non-hospital settings, and can be backed up by polymerase chain reaction tests during wave surges when the number of false-negative antigen tests increases.

As in the general population, Omicron has also caused a rapid rise in mild COVID-19 infections in healthcare workers. There are currently 1265 active healthcare worker infections in the Western Cape, with only one admission (WCGH). This is nearly $50 \%$ more staff infected than occurred at the peak of any previous wave. If each health worker has just two high-risk contacts among their colleagues, then $\sim 3795$ healthcare workers $-11 \%$ of the Western Cape Department of Health's employees - are currently off work as a result of quarantine or isolation (WCGH). Reports have surfaced in the past week from around the country and internationally - clinics and theatres are being closed due to staff shortages, emergency services are threatened. Healthcare for all patients will inevitably be compromised in the face of such extreme staff shortages.

Omicron has highlighted that current quarantine and isolation guidelines are counterproductive and must change to keep essential services operational. Given that most COVID-19 cases go undiagnosed, during a COVID-19 surge, exposure to asymptomatic cases is inevitable. Therefore, vaccination, ventilation and masking are our primary prevention tools. Isolating a small proportion of COVID-19 cases and quarantining their contacts does not protect individuals and may create a false sense of security if the public assumes that all COVID-19 cases and contacts are effectively in isolation or quarantine. The COVID-19 Ministerial Advisory Committee (MAC) has reportedly recommended that quarantining and contact tracing be abandoned. ${ }^{[11]}$ To maintain essential services, isolation periods for essential workers must also be shortened to allow those with minimal symptoms to return to work early with appropriate personal protective equipment. Finally, in preparation for future COVID-19 waves, essential workers should be prioritised for vaccines that provide the best protection against infection and offered boosters to ensure that protection against infection is maintained, when possible, in addition to preventing severe disease.

At the start of the pandemic, testing, quarantining, and isolation policies were our only tools to contain and mitigate COVID-19 in a completely susceptible population, and underpinned by a different understanding of transmission at that stage. Many people, including healthcare workers, have been traumatised by repeated waves of illness and mortality over the past 2 years. Changing strategies will inevitably provoke anxiety in those who still take comfort in the idea that COVID-19 can be isolated and eliminated. However, as COVID19 evolves, and we learn more about the pathogen, transmission, immunology, clinical course, treatment, and the effectiveness of prior interventions, so must our policies. Omicron is significantly more infectious than the wild type, and the mitigation of transmission from prior immunity is minimal. Nonetheless, after multiple waves of COVID-19 infection and vaccines, our bodies are better protected against severe disease, independent of any putative pathogenicity benefits of this variant. We must shift our monitoring away from cases towards hospitalisations. We cannot continue to cripple the healthcare system and the economy with what does not work overwhelming primary healthcare services with high-volume testing of mild COVID-19, quarantining contacts, and isolating cases for a prolonged period. People must stay at home if they are mildly ill, and only go to hospital if they are unwell and in need of treatment. Indoor masking, avoiding large indoor crowds, socialising outdoors, ventilation, and most importantly vaccination are the best defence against future seasons of endemic COVID-19, and the best way of ensuring that our health services are able to provide optimal management of all health conditions.

\section{Andrea S Mendelsohn (D), Angela de Sá}

Western Cape Government: Health, South Africa; Division of Family Medicine, School of Public Health and Family Medicine, Faculty of

Health Sciences, University of Cape Town, South Africa

andrea.mendelsohn@westerncape.gov.za

\section{Erna Morden}

Western Cape Government: Health, South Africa; Division of Public Health Medicine, School of Public Health and Family Medicine, Faculty of Health Sciences, University of Cape Town, South Africa

\section{Benjamin Botha}

Western Cape Government: Health, South Africa

\section{Andrew Boulle}

Western Cape Government: Health, South Africa; Division of Public Health Medicine, School of Public Health and Family Medicine, Faculty of Health Sciences, University of Cape Town, South Africa

\section{Masudah Paleker}

Western Cape Government: Health, South Africa; Division of Health Systems and Public Health, Department of Global Health, Faculty of Medicine and Health Sciences, Stellenbosch University, Cape Town, South Africa

\section{Mary-Ann Davies}

Western Cape Government: Health, South Africa; Division of Public Health Medicine, School of Public Health and Family Medicine, Faculty of Health Sciences, University of Cape Town, South Africa 
1. Viana R, Moyo S, Amoako DG, et al. Rapid epidemic expansion of the SARS-CoV-2 Omicron variant in southern Africa. MEDRXIV-2021-268028v1-deOliveira: (2021). https://www.krisp.org.za/ publications.php?pubid $=369$ (accessed 20 December 2021).

2. Western Cape Digital Press Conference. 15 December 2021. https://m.facebook.com/windealan/ videos (accessed 20 December 2021)

3. COVID-19 Public Vaccine Dashboard. https://sacoronavirus.co.za/latest-vaccine-statistics/ (accessed 20 December 2021).

4. Western Cape Digital Press Conference. 25 March 2021. https://m.facebook.com/windealan/videos (accessed 20 December 2021).

5. Cohen C, Kleynhans J, von Gottberg A, et al. SARS-CoV-2 incidence, transmission and reinfection in a rural and an urban setting: Results of the PHIRST-C cohort study, South Africa, 2020 - 2021. medRxiv 2021 (epub 22 July 2021). https://doi.org/10.1101/2021.07.20.21260855

2021 (epub 22 July 2021 ). https://doi.org/10.11 101/2021.07.20.21260855
6. Mistry DA, Wang JY, Moeser ME, Starkey T, Lee LYW. A systematic review of the sensitivity and Mistry DA, Wang JY, Moeser ME, Starkey T, Lee LYW. A systematic review of the sensitivity and
specificity of lateral flow devices in the detection of SARS-CoV-2. BMC Infect Dis 2021;21(1):828. specificity of lateral flow devices in the detection
https://doi.org/10.1186/s12879-021-06528-3

7. Brummer LE, Katzenschlager S, Gaeddert M, et al. Accuracy of novel antigen rapid diagnostics for Brummer LE, Katzenschlager S, Gaeddert M, et al. Accuracy of novel antigen rapid diagnostics for
SARS-CoV-2: A living systematic review and meta-analysis. PLoS Med 2021;18(8):e1003735. https:// SARS-CoV-2: A living systematic review
8. Woloshin S, Patel N, Kesselheim AS. False negative tests for SARS-CoV-2 infection - challenges and implications. N Engl J Med 2020;383(6):e38. https://doi.org/10.1056/NEJMp2015897

9. South African COVID-19 Modelling Consortium. COVID-19 modelling update: Consideration for a potential fourth wave 2021. https://www.sacmcepidemicexplorer.co.za (accessed 20 December for a pote.

10. GOV.UK. SAGE 99 minutes: Coronavirus (COVID-19) response, 16 December 2021. https:/ www.gov.uk/government/publications/sage-99-minutes-coronavirus-covid-19-response-16december-2021 (accessed 19 December 2021)

11. Fokazi S. SA could soon drop quarantining of Covid-19 contacts as this doesn't serve its purpose says MAC. Times Live, 19 December 2021. https://www.timeslive.co.za/news/south-africa/202112-19-sa-could-soon-drop-quarantining-covid-19-contacts-as-this-doesnt-serve-its-purpose-saysmac/ (accessed 20 December 2021).

S Afr Med J 2022;112(2):68-70. https://doi.org/10.7196/SAMJ.2022.v112i2.16348 